\title{
Guest Editorial Wideband CDMA I
}

$\mathbf{M}$ OBILE communications services are penetrating our society at an explosive rate. The major services that the second-generation mobile systems provide are limited to basic services (e.g., voice and low-bit rate data). We have entered the 21 st century, when demands for a variety of wideband services (e.g., high-speed Internet access and the video/high-quality images transmission) will continue to increase. The third-generation (3G) mobile systems (called IMT-2000) are being designed to support wideband services, at data rates as high as $2 \mathrm{Mb} / \mathrm{s}$, with the same quality as fixed networks. It is everyone's wish that wireless could act like a wired connection. To realize true IMT-2000 systems, a new wideband wireless access technology incorporating as many recent technology developments as possible is necessary.

Wideband direct sequence code division multiple access (wideband CDMA) is emerging as the predominant wireless access technology for the $3 \mathrm{G}$ systems, and is being developed throughout the world. Wideband CDMA is designed to flexibly offer wideband services, such as wireless Internet services (i.e., peak rate of $384 \mathrm{~kb} / \mathrm{s}$ to download information from the Web) and video transmissions (data rate up to $2 \mathrm{Mb} / \mathrm{s}$ ). Wideband is essentially about data rate. The physical limitations and impairments to radio channels (bandwidth constraints, multipath fading, noise, and interference) present a fundamental technical challenge to the goal of reliable high data rate communications.

The emphasis of this special issue is on advanced wireless wideband CDMA techniques. Wideband CDMA supports intercell asynchronous/synchronous operation, adaptive (variable rate) transmission, and applications of advanced techniques such as adaptive antenna arrays and turbo codes.

- One objective is cell acquisition for intercell asynchronous operation, which does not require interbase-station synchronization; therefore, there is no requirement on any external system such as GPS to allow for easy deployment in all types of environments.

- Adaptive (variable rate) transmission provides multimedia services. To achieve higher bit rates, multiple downlink physical channels (or multicode) can be transmitted in parallel in one direction.

- The adaptive antenna array directs beam nulls to interference sources to maximize the signal-to-interference ratio of each user. In the case of voice-only services, the appli-

Publisher Item Identifier S 0733-8716(00)06103-5. cation of the antenna arrays is rather impractical because a large number of antenna elements is required. However, antenna arrays are particularly useful for multimedia communications in which different users are transmitting at different data rates. High-rate users (Internet and video), although their numbers are not large, give significant interference to low-rate users (in particular, voice users); thus, without adaptive antenna array, the link capacity would be significantly reduced. In wideband CDMA, dedicated pilot symbols in both uplink and downlink facilitate userunique antenna patterns.

- Turbo coding has been shown to be extremely good for high-rate high-quality services because of its relatively large coding gain with reasonable computation complexity. Turbo codes are constructed by concatenating two or more recursive systematic codes. The main features of turbo coding are a) concatenating two or more recursive systematic codes, b) using a pseudorandom interleaver, and c) iterative decoding algorithm.

\author{
JIANGZHOU WANG, Guest Editor \\ University of Hong Kong \\ Hong Kong \\ FUMIYUKI ADACHI, Guest Editor \\ Tohoku University \\ Japan \\ Paul Walter BAiER, Guest Editor \\ University of Kaiserslautern \\ D-67663 Kaiserslautern, Germany \\ JAMES S. LEHNERT, Guest Editor \\ Purdue University \\ West Lafayette, IN 47907-1285 USA \\ WAYNE E. STARK, Guest Editor \\ University of Michigan \\ Ann Arbor, MI 48109 USA
}

MiCHAEL B. PURSLEY, J-SAC Board Representative

\section{ACKNOWLEDGMENT}

The Guest Editors would like to thank the authors for their submissions, the reviewers for their high-quality reviews, and Prof. M. B. Pursley for his advice. 


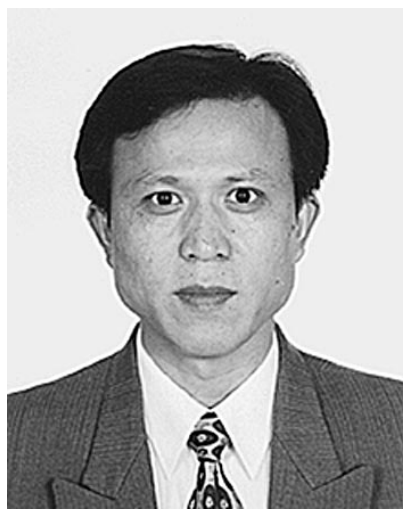

Jiangzhou Wang (M'91-SM'94) received the B.S. and M.S. degrees from Xidian University, Xian, China, in 1983 and 1985, respectively, and the Ph.D. degree (with Greatest Distinction) from the University of Ghent, Belgium, in 1990, all in electrical engineering.

From 1990 to 1992, he was a Postdoctoral Fellow with the University of California at San Diego, where he worked on the research and development of cellular CDMA systems. From 1992 to 1995, he was a Senior System Engineer at Rockwell International Corporation, Newport Beach, CA, where he worked on the development and system design of wireless communications. Since 1995, he has been with the University of Hong Kong, where he is currently an Associate Professor. He has been a Committee Member of a number of international conferences. He is teaching and conducting research in the areas of wireless mobile and spread spectrum communications.

Dr. Wang is an Editor for IEEE TRANSACTIONS ON COMMUNICATIONS and a Guest Editor for IEEE Journal on SELECTED AREAS IN COMMUNICATIONS for special issues on Wideband CDMA. He holds one U.S. patent in the GSM system.

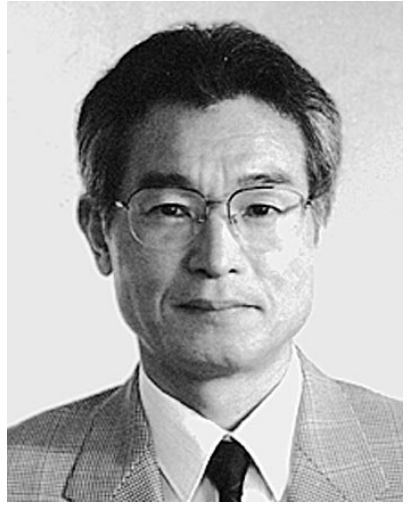

Fumiyuki Adachi (M'79-SM'90) received the B.S. and Dr.Eng. degrees in electrical engineering from Tohoku University, Sendai, Japan, in 1973 and 1984, respectively.

In 1973, he joined the Electrical Communications Laboratories of Nippon Telegraph \& Telephone Corporation (now NTT) and conducted various research related to digital cellular mobile communications. From July 1992 to December 1999, he was with NTT Mobile Communications Network, Inc., where he led a research group on wideband/broadband CDMA wireless access for IMT-2000 and beyond. Since January 2000, he has been at Tohoku University, Sendai, Japan, where he is a Professor in the Department of Electrical Communications at Graduate School of Engineering. From October 1984 to September 1985, he was a United Kingdom SERC Visiting Research Fellow in the Department of Electrical Engineering and Electronics at Liverpool University. From April 1997 to March 2000, he was a Visiting Professor at Nara Institute of Science and Technology, Japan. He has published more than 150 papers in journals and more than 60 papers in international conferences.

Dr. Adachi served as a Guest Editor of an IEEE Journal ON SELECTED AREAS IN COMMUNICATIONS special issue on Broadband Wireless Techniques (October 1999). He was a co-recipient of the IEEE TRANSACTIONS ON VEHICUlaR TeChNOLOGY Best Paper of the Year Award in 1980 and again in 1990. He is a Member of the Institute of Electronics, Information, and Communication Engineers of Japan (IEICE) and was a co-recipient of the IEICE Transactions Best Paper of the Year Award in 1996 and again in 1998.

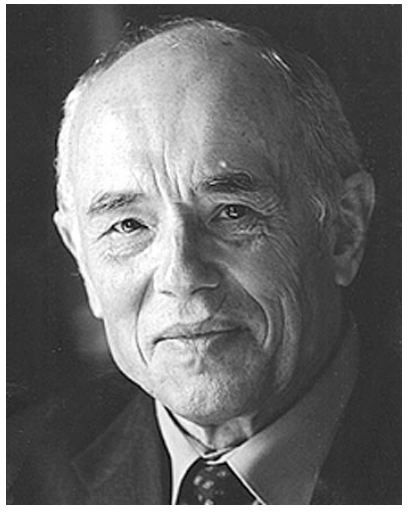

Paul Walter Baier (M'82-SM'87-F'95) was born in Backnang, Germany, in 1938. He graduated from the Technical University Munich, Germany, where, after graduation, he acted as a Senior Lecturer until 1970.

In 1970 he joined the Central Telecommunications Laboratories of Siemens AG, Munich, where he became Head of the Spread Spectrum Development Group and was engaged in various topics of communication engineering. Since 1973 he has been a Professor of Electrical Communications and Director of the Institute for RF Communications and Fundamentals of Electronic Engineering at the University of Kaiserslautern, Germany. His main research interests are spread spectrum techniques, impulse compression radars, imaging radars, mobile radio systems, and adaptive antennas. The basics of the TD-CDMA component of the UMTS Terrestrial Radio Access System (UTRA) adapted by ETSI in January 1998 and also forming part of the IMT-2000 standard agreed upon by 3 GPP were developed by him in cooperation with Siemens. He (co)authored two books and more than 100 papers and supervised more than

40 doctoral theses.

Dr. Baier is a Member of the U.R.S.I. Member Committee Germany. He was awarded the Innovation Prize of the Mannesmann Mobile Radio Foundation in 1999. 

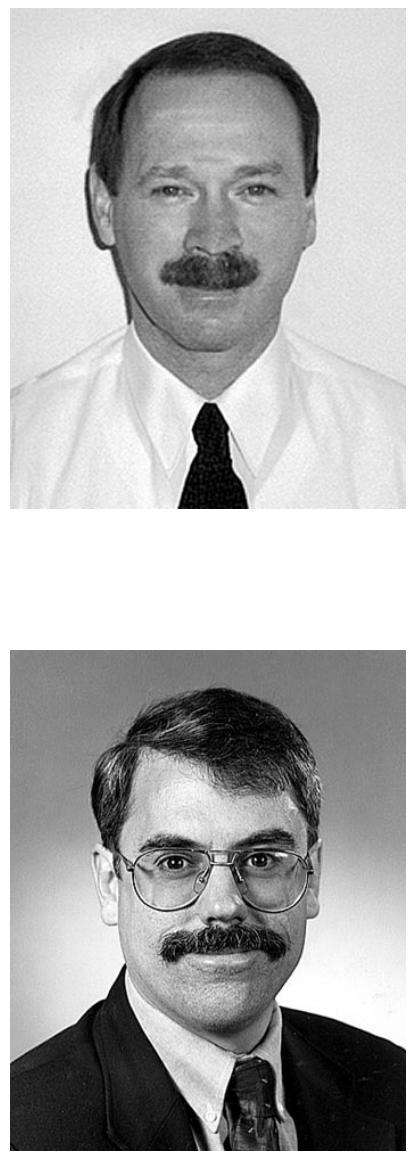
(MURI) project on Low Energy Mobile Communications. COMMUNICATIONS. 1982 respectively.
James S. Lehnert (S'83-M'84-SM'95-F'00) received the B.S. (Highest Honors), M.S., and Ph.D. degrees in electrical engineering from the University of Illinois at Urbana-Champaign in 1978, 1981, and 1984, respectively.

From 1978 to 1984, he was a Research Assistant at the Coordinated Science Laboratory, University of Illinois, Urbana. He was a University of Illinois Fellow from 1978 to 1979 and an IBM Pre-Doctoral Fellow from 1982 to 1984 . He has held summer positions at Motorola Communications, Schaumburg, IL, in the Data Systems Research Laboratory; and at Harris Corporation, Melbourne, FL, in the Advanced Technology Department. He is currently a Professor of Electrical and Computer Engineering at Purdue University, West Lafayette, IN.

Dr. Lehnert has served as Editor for Spread Spectrum for the IEEE TRANSACTIONS ON COMMUNICATIONS and as Guest Editor for the IEEE JOURNAL ON SELECTED AREAS IN

Wayne E. Stark (S'80-M'82-SM'94-F'98) received the B.S. (with highest honors), M.S., and Ph.D. degrees in electrical engineering from the University of Illinois, Urbana in 1978, 1979, and

Since September 1982, he has been a faculty member in the Department of Electrical Engineering and Computer Science at the University of Michigan, Ann Arbor, where he is currently Professor. His research interests are in the areas of coding and communication theory, especially for spread-spectrum and wireless communication networks.

Dr. Stark is a member of Eta Kappa Nu, Phi Kappa Phi, and Tau Beta Pi. From 1984 to 1989, he was Editor for Communication Theory of the IEEE TRANSACTIONS ON COMMUNICATIONS in the area of Spread-Spectrum Communications. He was involved in the planning and organization of the 1986 International Symposium on Information Theory which was held in Ann Arbor. He was selected by the National Science Foundation as a 1985 Presidential Young Investigator. He is Principal Investigator of a Army Research Office Multidisciplinary University Research Initiative 